\title{
Infective Endocarditis: A Growing Concern and Treatment Approaches: An Observational Study
}

\author{
Muhammad Daoud Butt*,1, Muhammad Sarfraz Nawaz', Basit Ramazan², Tooba Malik ${ }^{1}$, Sidra Sulehri ${ }^{3}$ \\ ${ }^{1}$ Department of Pharmacy, Quaid-i-Azam University, Islamabad, Pakistan.
}

${ }^{2}$ School of Pharmaceutical Sciences, University Sains Malaysia, Malaysia.

${ }^{3}$ Department of Pharmacy, Pakistan Institute of Medical Sciences, Islamabad, Pakistan.

\begin{abstract}
Objective: To determine the frequency of prevailing microorganism, treatment approach, different treatment complexities and rationalize the approach for treating Infective Endocarditis (IE) patient.

Material and Methods: The retrospective study was conducted from 01st January 2015 to 31st October 2019. Data was collected and after applying exclusion criterion 40 patients were selected and their available record was evaluated. To obtain the conclusive results, frequency and percentages were calculated.

Results: The mean age of patient in studied group was 34.02 years with standard deviation (S.D) 13.02 years, and $65 \%$ of patients included were male and 35\% were female. Staphylococcus aureus (S.aureus) was the prevalent microorganism found in most cases i.e; $60 \%$. Mitral valve was mostly affected; Rheumatic heart disease was the leading factor of IE in patients. Most of the patients complained of shortness of breath and high-grade fever. Benzylpenicillin and aminoglycosides were most commonly prescribed for patients. The medication health care cost was on the higher side at about $1038 \mathrm{PKR} /$ day considering all healthcare facilities provided free by the government.
\end{abstract}

Conclusion: In most of the younger population active infective endocarditis remains the most prevailant disease. Rheumatic heart disease remains to be the most common underlying factor for heart disease. Overall treatment cost of therapy was found to be high due to vigorous antibiotic therapy.

Keywords: Infective endocarditis, Microorganism, Rheumatic heart disease, Medication treatment, Vegetation, Staphylococcus aureus.

\section{INTRODUCTION}

Infective Endaocarditis (IE) is a systemic atypical disease, it has several different forms and its symptoms are not constant all over (linked particularly with clinical findings, any cardiovascular complications, the microbiological investigations, the manifestation of other complications, and patient characteristics); vascular endothelium is mostly affected by the lesion and vegetation. Heart valves are frequently affected by IE, which ultimately causes local destruction of valves, along with regurgitation problems. The estimated reported incidence annually ranges between 5.0-7.9 cases IE /100,000 people. The disease prevalance ratio among male and female is $2: 1$ respectively $[1,2]$. In Infective endocarditis the most apparent features associated with it are age, preexisting degenerative valvular lesions, the presence of a prosthetic valve or a prosthetic substance present in cardiac valve restoration, preceding infective endocarditis, certain congenital heart defects, hemodialysis, co-morbidities for instance diabetes mellitus, human immunodeficiency virus(HIV) infection, intravenous substance abuse [3].

*Address correspondence to this author at the Department of Pharmacy, Quaid-i-Azam University Islamabad, Pakistan.

E-mail: muhammaddaoud10@yahoo.com
Problems associated with IE comprise congestive heart failure, unrestrained infection, venous embolism, end-stage renal disease, myocarditis, pericarditis and rheumatic problems. The cardiac arrest seems after acute regurgitation triggered by acute valve demolition. Persistent uncontrolled infection is usually due to resistant microorganisms. The left sided infective endocarditis with choronic embolism usually designated as Stroke. In $20-50 \%$ of patients pulmonary embolism appears oftenly due to native right-sided and pacemaker that leads to Infective endocarditis $[4,5]$.

Clinical presentations are the main tools for identification of infective endocarditis, vegetation identified by echocardiographic and microbiological identification (blood cultures, histological, molecular biology techniques) [6]. To clarify the existence of vegetation, valvular regurgitation, paravalvular abscesses and very often dehiscence and fistulae Transthoracic echocardiography (TTE) associated with transesophageal echocardiography (TEE) technique is used [4].

Earlier infective endocarditis with rheumatic valve disease was recognized as a disease of young population; presently. Due to increase in invasive diagnostic procedures like prosthetic valve replacement, pacemakers etc; it begain to affects older patients as well $[4,7,8]$. 


\section{MATERIALS AND METHODS}

In this retrospective review of existing clinical records available at Tertiary care hospital of Islamabad. The hospital had specialized cardiac center where all patients were treated and all procedures were done. All the infective patients who have ages ( $>10$ years) were included. The total of 134 files with a search of infective endocarditis were obtained through hospital database. Only 40 cases with confirmed infective endocarditits diagnosis were selected.

A case was demarcated as any mature patient admitted with a ruling of 'definite' IE ashored on the amended Duke's criteria [9].

The facts collected during the study from all cases were: age, sex, past of heart disease, techniques, and conditions at risk for IE, echocardiographic conclusions, non-cardiac co-morbidities,preadmission use of antibiotic, presenting symptoms, difficulties, microbiological data.

The exclusion criteria used to evaluate cases were: Patient with age below 10, patients with any kind of intravenous drug abuse, and suspected cases of infective endocarditis.

Permission from the institute "Ethical Review Board" has been obtained for the study. The statistical tool used to evaluate the data was Microsoft Excel 2016. The study will give us a brief overview of the different aspects of Infective Endocarditis. Being descriptive data only percentages were calculated.

\section{RESULTS}

The present study consists of 40 cases out of which $26(65 \%)$ men and 14 (35\%) women; overall mean age was $34.025 \pm 18$ (years). There were $30(75 \%)$ patients under 35years (range 12-35); the others were above 35 years of age (range 36-70).

The aortic valve was infected in $5(12.5 \%)$ cases, the mitral valve was infected in $8(40 \%)$. Culture sensitivity test showed there were $12(30 \%)$ cases with Methicillin-Resistant Staphylococcus aureus, 8(20\%) cases were of Streptococcus Viridian group, 5(12.5\%) cases were of Escherichia coli and $15(37.5 \%)$ cases showed culture-negative which was due to self-medication of antibiotic before hospitalization.

The majority of the patients were having Rheumatic heart disease as primary underlying disease $(\mathrm{n}=23 ; 53 \%)$. The site of anatomic involvement was as follows: majorly mitral valve in $14(35 \%)$ cases and minorly aortic valve in $5(12.5 \%)$ cases as evident from Table $\mathbf{1}$.
Table 1. Predisposing Conditions.

\begin{tabular}{|l|c|}
\hline Underlying Cardiac Diseases & No. of Patients (\%) \\
\hline Rheumatic Heart Disease & $23(57.5)$ \\
\hline Mitral valve disease & $14(35)$ \\
\hline Aortic valve disease & $5(12.5)$ \\
\hline Mixed valvular heart disease & $4(10)$ \\
\hline Congenital Heart Disease & $11(27.5)$ \\
\hline Bicuspid aortic valve & $6(15)$ \\
\hline Atrial septal defect & $2(5)$ \\
\hline Ventricular septal defect & $2(5)$ \\
\hline Tetralogy of Fallot & $1(2.5)$ \\
\hline Non-Cardiac Diseases & $6(15)$ \\
\hline Chronic kidney disease & $3(7.5)$ \\
\hline Inflammatory bowel disease & $1(2.5)$ \\
\hline Dental procedure & $2(5)$ \\
\hline
\end{tabular}

All Patients received on average 12.3 days of multi-drug antibiotic treatment before either completion of treatment or being discharged with prescribed medication.

Different treatment combinations were seen of which prominent were, Benzylpenicillin with aminoglycosides (gentamy$\mathrm{cin} /$ amikacin) was the commonly used antibiotic and used in $16(40 \%)$ cases. Other antibiotics used were vancomycin $10(25 \%)$, ceftriaxone $12(30 \%)$ and fluoroquinolones $2(5 \%)$ $[10,11]$.

The characteristics of the patients with active IE were noted. Incidences of high-grade fever, shortness of breath and chest palpatation were significantly observed symptoms as evident from Table 2.

Table 2. Frequency of Chief Complication.

\begin{tabular}{|l|c|c|}
\hline Chief Complication & Frequency & Percentage (\%) \\
\hline Shortness of Breath & 12 & 30 \\
\hline High-Grade Fever & 9 & 22.5 \\
\hline Productive Cough & 3 & 7.5 \\
\hline Chest Discomfort & 7 & 17.5 \\
\hline Vertigo & 5 & 12.5 \\
\hline Periorbital Swelling & 1 & 2.5 \\
\hline Afebrile & 2 & 5 \\
\hline Immobilize Left Arm & 1 & 2.5 \\
\hline
\end{tabular}


There were no HIV-positive patients and no drug abusers. Patients in which there was negative culture obtained the antibiotic treatment consisted of Vancomycin + Gentamycin + Ceftriaxone whereas mostly the empirical therapy usually consisted of Benzylpenicillin + Gentamycin + Co-Amoxicillin.

Patients selected for the study were receiving antibiotic therapy for Infective Endocarditis along with supportive therapy, according to Hospital's protocols and patient symptoms, which include mainly Diuretics (Furosemide and Spironolactone), Beta-blockers, Calcium channel blockers Statins, and Anticoagulants.

Supportive therapy is of important concern for maintaining and optimizing the patients' health, but this also leads to extravagant prescribing and so results in polypharmacy. Problems of Drug interactions became more visible due to this polypharmacy. As in the above cases, $70 \%$ of patients were receiving Furosemide as supportive therapy for maintaining B.P but its irrational prescribing can lead to nephrotoxicity and auditory toxicity for I.E patients as they have to take Gentamicin, Vancomycin or both for treatment. This Furosemide also causes hypokalemia and to overcome this, dose should be reduced instead of prescribing Potassium supplements.

The approximate medication cost that was found out during this retrospective review was about $1038 \mathrm{PKR} /$ day which was quite high keeping in view the health budget of Pakistan. The health care cost of infective endocarditis in the US is also increasing with evolving infection and reached up to 122,204 USD/15.3 days [11].

\section{DISCUSSION}

Infective endocarditis is a growing disease in our public. Prominent drifts have been witnessed in this study. Predominance in male is the major aspect and has been reported in another study [12-14]. Most of the studies and data available are western. In our general public, the cause behind male high proportion seems to be associated to socioeconomic bias as reported by researchers in their study [15].

The study also showed that the infective endocarditis predominance in males is on a higher side (55\%) contrary to the females that is just (45\%) as shown in Fig (1).

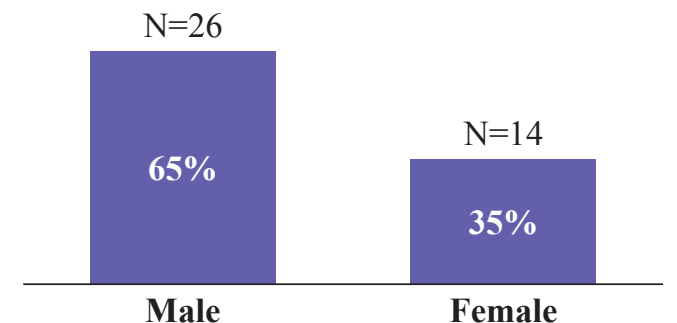

Fig. (1). Comparison of Sex with Definite Infective Endocarditis.
According, to a survey conducted by WHO the overall ratio of infection in male to female is $2: 1$ respectively but this study and also previous published data shows that most of the male population is affected with infective endocarditis with higher ratio as compared to WHO results [16].

Most of the patients selected for study under the designed criteria fit into the younger age cluster 10-30years as evident from Fig. (2).

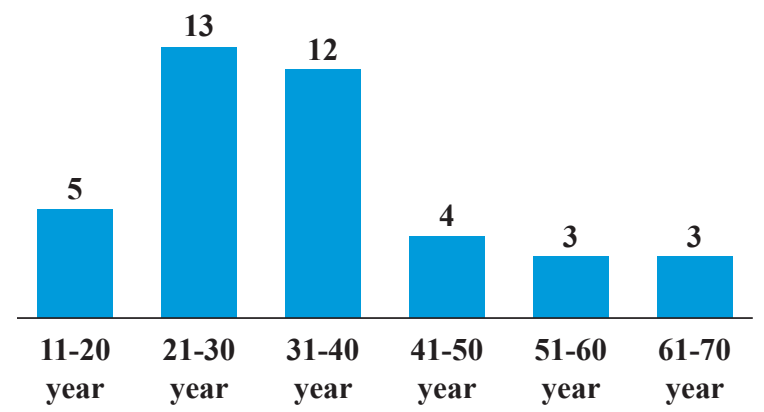

Fig. (2). Comparison of Infective Endocarditis in Different Age Groups.

The reason to support this outcome is grounded on the statement, the predominanent prevalence of chronic rheumatic heart disease in developing world and in younger age clusters which make them inclined to infective endocarditis [17]. On the other hand, improved healthcare system and technological advancement in developed countries have not only given escalation to prompting elements like deteriorating valvular disease, prosthetic valves placement but also augmented exposure to nosocomial bacteremia. According to study the usual age cluster who got infective endocarditis is 23-39 years.

In this study, the major causative organism is Staphylococcus aureus and has a prevalence rate of $60 \%$. Whereas the other commonly found organisms belongs to Streptococcus viridian group which infects about $20 \%$ of the patients as shown in Table 3.

Table 3. Culture Sensitivity Results in Comparison among Cases.

\begin{tabular}{|l|c|c|}
\hline Causative Organism & No. of Patients & Percentage \\
\hline Escherichia coli & 5 & $12.5 \%$ \\
\hline $\begin{array}{l}\text { Methicillin-resistant } \\
\text { Staphylococcus aureus } \\
\text { MRSA }\end{array}$ & 12 & $30 \%$ \\
\hline Streptococcus viridian & 8 & $20 \%$ \\
\hline Negative & 15 & $37.5 \%$ \\
\hline
\end{tabular}

There is a changing trend which is usually seen in the causative organism of infective endocarditis [18]. According to a previous cohort study, it is also evident that the most 
prevailing organism for infective endocarditis is S.aureus as documented by Vance et al. S aureus remains the most common reason of developing Healthcare-associated infection leading to IE (218/1779 patients, 39.1\%), accounting for $25.9 \%$ (Australia/New Zealand) to $54.2 \%$ (Brazil) of cases [19]. In this case study, $20 \%$ of the patients with negative culture are showing comprehensive signs of infective endocarditis. This high culture negativity is understandable and is most likely due to unjustified use of antibiotics before admission in specialized health care i.e., Cardiac hospital and also, due to inappropriate standards of diagnostic laboratory which carried out culture sensitivity tests. Correspondingly, in this study, the important association is established between the prior intake of antibiotics and culture negative endocarditis.

The special procedures is to be used for the identification of pathogen in culture-negative disease. In such a situation, the other diagnostic tests such as indirect fluorescence, ELISA, complement fixation, serology, agglutination and polymerase chain reaction (PCR) can be used for definitive diagnosis [20]. Major problem with culture negative IE is that it usually does not respond to the empirical treatment. Molecular results can go on positive side even after continuing antimicrobial therapy.

Previous studies reported data like presenting symptoms, clinical features and laboratory findings were comparable to this study. Previous studies also showed the predominance of mitral valve involvement in the vegetation of the organism. $40 \%$ of patients have MVR and showed signs of vegetation on the Mitral valve when Echo was done [21].

This extravagant prescribing practice also increased the economic burden on health. This can be minimized with proper evaluation of a patient for symptoms before prescribing the drugs and prescription monitoring by a pharmacist for any significant interactions.

\section{CONCLUSION}

There is persistent evolution of epidemiology of IE seen in individualized states, the features which remained qualitatively the same as of initial valve lesions that stimulate bacterial colonization. The study also indicated that there will be a need to rationalize our health care approach to treat infective endocarditis. The growing concern is the continuously increasing rate of infection due to resistant strains of bacteria. Self-medication is also seen as a growing concern, increase in such cases there is a negative culture sensitivity test which is problematic on part of the physician to rationalize the medication. This demands a vigorous antibiotic treatment using broad-spectrum antibiotics.

The sociocultural features brings modifications and variations in epidemiology of disease with factors such as, aging of the population, rise in the number of patients equipped with intracardiac implants, amplified use of intravenous recreation- al drugs and escalations of bacteremia associated with healthcare-related invasive manipulations. Such favourable circumstances allow skin pathogens (for example, Staphylococcus spp.) and enteral streptococci to cause Infective endocarditis rather than more-classical oral streptococci.

The younger people in the community remains the most affected population with Infective endocarditis. The major underlying heart disease continues to be Rheumatic heart disease. Isolation of causative micro-organism is an essential part of the diagnosis of IE for appropriate antimicrobial therapy. Patients with a past of cardiac surgery and individuals with neurologic and embolic complications had a poorer prognosis. Higher incidence of IE in men is likely to reflect the social factor. Changing trends as seen in this study demands further advanced studies and necessary evaluation.

\section{FUNDS FOR STUDY}

Declared none.

\section{CONFLICT OF INTEREST}

Declared none.

\section{ACKNOWLEDGEMENTS}

We thank all authors and who help and guide through the manuscript.

\section{AUTHORS' CONTRIBUTION}

- Muhammad Daoud Butt (Data Collection, Analysis, Study design, Write up, Results interpretation).

- Muhammad Sarfraz Nawaz (Analysis, data interpretation).

- Tooba Malik (Data Collection, Write up and reference).

- Basit Ramazan (Write up, analysis).

- Sidra Sulehri (Supervision, Results interpretation).

\section{REFERENCES}

[1] DeSimone DC, Tleyjeh IM, De Sa DDC, et al. Incidence of infective endocarditis caused by viridans group dtreptococci before and after publication of the 2007 american heart association endocarditis prevention guidelines. Circulation 2012; 126(1): 60-4.

DOI: $10.1161 /$ CIRCULATIONAHA.112.095281

[2] Duval X, Delahaye F, Alla F, et al. Temporal trends in infective endocarditis in the context of prophylaxis guideline modifications: Three successive population-based surveys. J Am Coll Cardiol 2012; 59(22): 1968-76.

DOI: 10.1016/j.jacc.2012.02.029

[3] Sy RW, Kritharides L. Health care exposure and age in infective endocarditis: Results of a contemporary population-based profile of 1536 patients in Australia. Eur Heart J 2010; 31(15): 1890-7. DOI: 10.1093/eurheartj/ehq110

[4] Selton-Suty C, Célard M, Le Moing V, et al. Preeminence of 
Staphylococcus aureus in infective endocarditis: A 1-year population-based survey. Clin Infect Dis 2012; 54(9): 1230-9. DOI: $10.1093 / \mathrm{cid} / \mathrm{cis} 199$

[5] Sonneville R, Mirabel M, Hajage D, et al. Neurologic complications and outcomes of infective endocarditis in critically ill patients: The endocardite en reAnimation prospective multicenter study. Crit Care Med 2011; 39(6): 1474-81. DOI: 10.1097/CCM.0b013e3182120b41

[6] García-Cabrera E, Fernández-Hidalgo N, Almirante B, et al. Neurologic complications of infective endocarditis: Risk factors, outcome, and impact of cardiac surgery: A multicenter observational study. Circulation 2013; 127(23): 2272-84. DOI: 10.1161/CIRCULATIONAHA.112.000813

[7] Topcuoglu MA, Kursun O, Buonanno FS, Isselbacher EM, Hurtado RM, Singhal AB. Ischemic and hemorrhagic stroke in infective endocarditis: Features, predictors and trends over three decades. Stroke 2017; 48: A84.

[8] McElhinney DB, Benson LN, Eicken A, Kreutzer J, Padera RF, Zahn EM. Infective endocarditis after transcatheter pulmonary valve replacement using the melody valve. Circ Cardiovasc Interv 2013; 6(3): 292-300.

DOI: 10.1161/CIRCINTERVENTIONS.112.000087

[9] Fournier P, Casalta J, Habib G, Messana T, Raoult D. Modification of the diagnostic criteria proposed by the Duke Endocarditis Service to permit improved diagnosis of Q fever endocarditis. Am J Med 1996; 100(6): 629-33.

DOI: $10.1016 / \mathrm{S} 0002-9343(96) 00040-\mathrm{X}$

[10] Snygg-Martin U, Gustafsson L, Rosengren L, et al. Cerebrovascular complications in patients with left-sided infective endocarditis are common: A prospective study using magnetic resonance imaging and neurochemical brain damage markers. Clin Infect Dis 2008; 47(1): 23-30. DOI: $10.1086 / 588663$

[11] Bor DH, Woolhandler S, Nardin R, Brusch J, Himmelstein DU. Infective endocarditis in the US, 1998-2009: A nation- wide study. PloS One 2013; 8(3): e60033.

DOI: 10.1371 /journal.pone.0060033

[12] Ruttmann E, Willeit J, Ulmer H, et al. Neurological outcome of septic cardioembolic stroke after infective endocarditis. Stroke 2006; 37(8): 2094-9.

DOI: 10.1161/01.STR.0000229894.28591.3f

[13] Kothari S, Ramakrishnan S, Bahl V. Infective endocarditis-an Indian perspective. Indian Heart J 2005; 57(4): 289.

[14] McKay G, Bunton R, Galvin I, Shaw D, Singh H. Infective endocarditis--a twelve year surgical outcome series. N Z Med J 2002; 115(1150): 124-6.

[15] Rehman S, Shabbier G, Shahid M, Shahid M. Clinical presentation of infective endocarditis. J Postgrad Med Inst 2011; 16(1): 55-63.

[16] Gupta A, Gupta A, Kaul U, Varma A. Infective endocarditis in an Indian setup: Are we entering the 'modern'era? Indian J Crit Care Med 2013; 17(3): 140. DOI: 10.4103/0972-5229.117041

[17] Rizvi S, Khan M, Kundi A, Marsh D, Samad A, Pasha O. Status of rheumatic heart disease in rural Pakistan. Heart 2004; 90(4): 394-9. DOI: 10.1136/hrt.2003.025981

[18] Khan NU, Farman MT, Sial JA, Achakzai AS, Saghir T, Ishaq M. Changing trends of infective endocarditis. J Pak Med Assoc 2010; 60(1): 24-7.

[19] Fowler VG, Miro JM, Hoen B, et al. Staphylococcus aureus endocarditis: A consequence of medical progress. JAMA 2005; 293(24): 3012-21. DOI: 10.1001/jama.293.24.3012

[20] Liesenfeld O, Lehman L, Hunfeld K-P, Kost G. Molecular diagnosis of sepsis: New aspects and recent developments. Eur J Microbiol Immunol (Bp) 2014; 4(1): 1-25.

DOI: 10.1556/EuJMI.4.2014.1.1

[21] Roberts WC, Ko JM. Some observations on mitral and aortic valve disease. Proc (Bayl Univ Med Cent) 2008; 21(3): 282-99. DOI: 10.1080/08998280.2008.11928412 